\title{
Control de movimiento robótico con detección cognitiva y facial mediante Emotiv EEG
}

\author{
Robotic motion control with cognitive and facial detection via Emotiv EEG
}

\author{
Sebastián Monge Lay ${ }^{1} \quad$ Diego Aracena Pizarro ${ }^{1}$ \\ Recibido 1 de diciembre de 2014, aceptado 11 de mayo de 2015 \\ Received: December 1, 2014 Accepted: May 11, 2015
}

\begin{abstract}
RESUMEN
Este trabajo presenta el desarrollo de una interfaz cerebro-computador utilizando el dispositivo Emotiv EEG, en donde se realizan reconocimientos de gestos faciales y pensamientos cognitivos, los que serán traducidos en movimientos que serán ejecutados por un robot Lego Mindstorms, con la idea de verificar si realmente Emotiv realiza un buen reconocimiento y puede ser utilizado para el movimiento de dispositivos. El trabajo es motivado por otras investigaciones realizadas, en donde se aplicaron distintas técnicas para el reconocimiento, pero en algunos casos la velocidad y en otros la precisión no fueron las esperadas, por lo que en esta investigación utiliza otros métodos para evitar esos problemas. Se realizan pruebas de reconocimiento con seis personas, arrojando en el reconocimiento cognitivo un $81 \%$ de precisión con dos acciones aprendidas y para el reconocimiento facial una precisión de $67 \%$ con seis gestos utilizados. Posteriormente se realiza una prueba en el sistema implementado con una arquitectura dirigida por eventos, junto con el dispositivo Emotiv y el robot, obteniendo una precisión del 70\%, evaluada según lo que el usuario quería hacer y lo que ejecutaba el robot, donde se muestra que el dispositivo Emotiv es recomendable para su uso en el control de movimientos, ya que entrega buenos resultados de reconocimiento y permite múltiples acciones en tiempo real.
\end{abstract}

Palabras clave: Emotiv, robot, EEG, electroencefalograma, señales mentales, gestos faciales, interfaces cerebro-computador BCI.

\section{ABSTRACT}

This papers presents the development of a brain-computer interface using the Emotiv EEG device, where a facial gestures and cognitive thought are detected, which will be translated into movements by a Lego Mindstorms robot. This translation is made to verify if the Emotiv is able to perform a good recognition and it can be used to control the movement of devices. This research work was motivated by previous works, where different recognition techniques were applied, but in some cases the speed and in others the precision of detection were not the expected. Therefore, in this research work uses others methods to solve these problems. Recognition tests were performed with 6 persons, resulting on an $81 \%$ of precision with cognitive recognition for two actions learned and a $67 \%$ of precision with facial recognition for six gestures used. Lately, a test was performed in a system implemented under an event-driven architecture, using the Emotiv device and the Lego robot, obtaining a precision on detection of $70 \%$, evaluated by comparison between the action desired by the user and the action effectively performed by the robot, which shows that the Emotiv device is recommendable for its use, given the quality/price factor, because it gives good recognition results and allows multiple actions in real time.

Keywords: Emotiv, robot, EEG, electroencephalogram, mental signal, facial gestures, brain-computer interfaces, $B C I$.

${ }^{1}$ Escuela Universitaria de Ingeniería Industrial, Informática y Sistemas. Universidad de Tarapacá. Casilla 6-D. Arica, Chile.

E-mail: smonge@live.cl; daracena@uta.cl 


\section{INTRODUCCIÓN}

Hoy la innovación en interfaces humano-computador está siendo muy frecuente en la industria, empezando con la consola de juegos Wiii de propiedad de la empresa Nintendo®, con su revolucionario mando inalámbrico que lee movimientos gracias a su sensor integrado de tres ejes [1], por otra parte la Kinect ${ }^{\circledR}$ para Xbox 360® de Microsoft ${ }^{\circledR}$, donde la interfaz es tu propio cuerpo gracias a su cámara RGB y su sensor de profundidad [2], que detectan los movimientos, entre otros dispositivos en el mercado.

Estas interfaces utilizan la movilidad del cuerpo para la detección, el cual no es el caso de Emotiv EEG® de Emotiv ${ }^{\circledR}$, que consiste en un casco neuronal de alta resolución con 14 canales y un giroscopio de dos ejes. El dispositivo es inalámbrico y se comunica en radiofrecuencia a una entrada USB del computador, que recibe las señales para su procesamiento [3], lo que permite mediante un ambiente desarrollado obtener las acciones cognitivas, afectivas y expresivas generadas por el usuario y transformarlas en alguna acción a realizar, como puede ser el movimiento de algún dispositivo. En la Figura 1 se puede apreciar el casco.

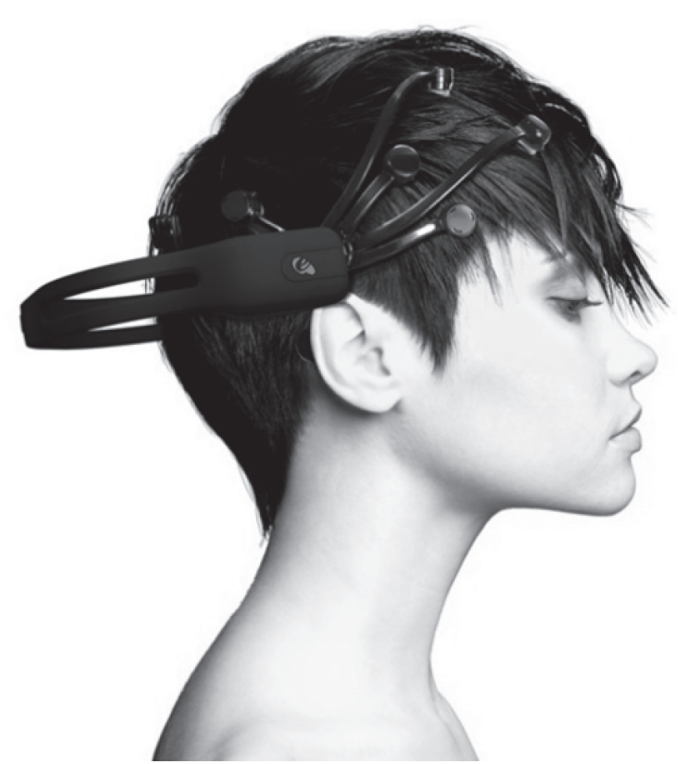

Figura 1. Dispositivo Emotiv EEG.

Existen diversos trabajos en torno a las interfaces cerebro-computador, como por ejemplo el control de un brazo robótico mediante electroencefalografía (EEG) [4], el que utiliza la tecnología Emotiv para controlar el brazo, pero en vez de emplear su software de reconocimiento, utiliza una implementación propia, logrando reconocer más acciones mentales que el propio Emotiv (que posee un máximo de cuatro), pero con una precisión bastante baja (menos del $40 \%$ de aciertos). Otro caso interesante es el control de una silla motorizada con un brazo mecánico incrustado, el que es controlado por medio de las señales obtenidas por un electroencefalograma, que fue construida para satisfacer las necesidades de las personas con movilidad reducida [5], pero su sistema era muy lento, debido a que se mostraba una pantalla con varias opciones de movimiento, la que se iluminaba por sectores cada cierto tiempo, y se detectaba la señal mental que se generaba cuando se iluminaba el sector que el usuario quería ejecutar, lo que consumía mucho tiempo, y podría ser riesgoso en el caso de utilizarlo en una silla de ruedas eléctrica y encontrarse con un desnivel o escaleras, y tener que esperar a que se ilumine la opción de parar para lograr detenerla. Por último y semejante a este trabajo es el control mental de un robot Lego Mindstorms ${ }^{\circledR}$ por medio del sistema Neurosky® [6] que es similar al Emotiv EEG, pero cuenta con un único canal de recepción de señales EEG y solo realiza detecciones de estados mentales (calma, concentración, etcétera) y no de pensamientos y gestos faciales como Emotiv.

Este trabajo se motiva en las investigaciones descritas anteriormente, tratando de solventar las falencias mencionadas, en búsqueda de un reconocimiento rápido y preciso, por lo que se desarrolla una interfaz de movimiento neuronal para el robot Lego Mindstorms, que es utilizado como dispositivo de movimiento, para medir su precisión mediante las diferentes acciones captadas por el casco de Emotiv, las que se traducirán en movimientos que serán ejecutados por el robot, con el fin de determinar qué tan cercano y efectivo es el reconocimiento, centrándose en acciones faciales (pestañear, masticar, etcétera) y cognitivas (pensamientos) para las pruebas.

\section{METODOLOGÍA}

Para la selección del dispositivo EEG se consideraron diversos factores como su accesibilidad, costo, portabilidad y cantidad de canales, siendo la mejor opción el dispositivo Emotiv EEG, que es un sistema acorde a las necesidades del proyecto y además es una decisión apoyada por el trabajo 
descrito en [7], que realiza una comparación con diversos dispositivos EEG, obteniendo la misma conclusión, sobre todo lo que más se destaca de Emotiv es su calidad/precio, además de ser un dispositivo portable, en comparación con los grandes sistemas de EEG que cuentan con muchos canales y grandes equipos de recepción de la señal.

En el caso de la selección del robot Lego Mindstorms es un robot armable, con tres servomotores, por lo que es ideal su utilización para medir los movimientos que serán generados por el casco, ya que su forma depende de cómo uno lo arme, y en este caso se optó por un diseño de dos ruedas con un motor independiente por cada una, como se observa en la Figura 2.

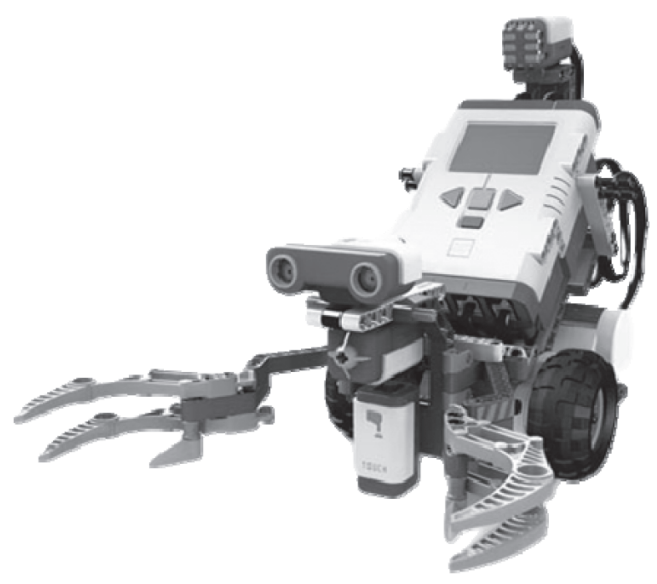

Figura 2. Robot Lego Mindstorms.

Posteriormente para las pruebas, se realizaron mediciones de reconocimiento facial y cognitivo (mental), en sujetos de prueba, para determinar los niveles de precisión, y así determinar si realmente Emotiv EEG se puede utilizar para realizar movimientos de dispositivos en tiempo real y con una alta precisión.

\section{EMOTIV EEG}

Emotiv EEG es un producto ofrecido por la empresa Emotiv que consiste en un dispositivo que realiza un electroencefalograma de alta resolución con 14 canales ubicados y nombrados según el sistema internacional 10-20 [8] más dos canales de referencia, posee un giroscopio de dos ejes, es inalámbrico y tiene una batería para 12 horas de uso continuo, se comunica con el computador por medio de un receptor USB que recibe las señales y las despacha a su software de detección que reconoce expresiones faciales, estados anímicos y pensamientos cognitivos, estas además pueden ser procesadas por medio del SDK, que está disponible para diferentes plataformas de programación [3].

La distribución de los sensores del Emotiv EEG como se muestra en la Figura 3 están posicionados de tal manera de obtener beneficios óptimos para la interacción hombre-máquina. Los sensores se encuentran principalmente en la corteza frontal, por lo que es útil para detectar gestos faciales (como el parpadeo) y también determinar ciertos tipos de ondas mientras el usuario se concentra en alguna tarea. Los sensores en P3(CMS) y P4(DLR) son los utilizados como referencia.

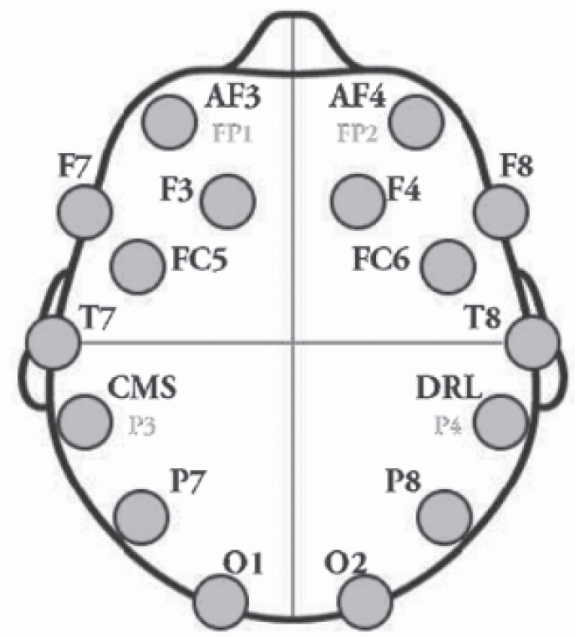

Figura 3. Distribución de sensores en Emotiv.

\section{ARQUITECTURA DEL SISTEMA}

Para la implementación del sistema se ha utilizado C\# como lenguaje de programación, el API de Emotiv para trabajar con el Emotiv EEG, el framework AForge.NET [9] para el desarrollo de la aplicación que realiza la conexión con el Robot Lego Mindstorms y un computador con sistema operativo Windows 8.1.

En lo que respecta a hardware se ha utilizado un computador con conexión bluetooth incorporada, que actúa como intermediario (unidad de procesamiento), con el dispositivo Emotiv EEG, el que se comunica con el computador usando su propio sistema inalámbrico con un adaptador USB y el robot 
Lego Mindstorms NXT que utiliza bluetooth para la comunicación [10]. En la Figura 4 se presenta la arquitectura de hardware utilizado.
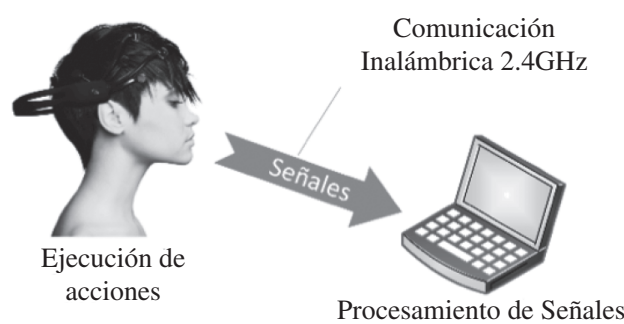
acciones
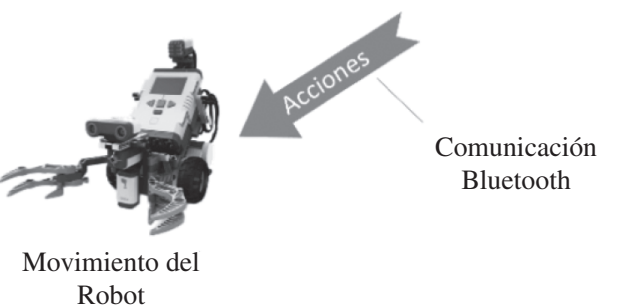

Figura 4. Arquitectura del sistema.

Respecto del software desarrollado, la Figura 5 muestra el diagrama de clases, donde se exponen a grandes rasgos los métodos implementados para el funcionamiento del sistema, destacando que el API de Emotiv (para C\#) denotado por la clase "EmoEngine", utiliza una arquitectura dirigida por eventos, por lo que la clase principal de la aplicación llamada "Robot" también lo implementa. Este no es el caso del API para manejar el robot Lego representado por la clase NXTBrick, que se crea una instancia de él (en la clase Robot), y se llama a sus funciones para establecer acciones en el robot.

En la Figura 6 se muestra el diagrama de secuencia que inicia el proceso de generación de eventos cuando se produce alguna señal en el Emotiv EEG, esta función se debe ejecutar constantemente para que analice en tiempo real la información otorgada por el casco sin procesar, la que al realizar la detección de alguna acción, genera eventos para que sean manejados por sus suscriptores. Esta se genera un proceso aparte, para no interrumpir al hilo principal.

En la Figura 7 se aprecia el diagrama de secuencia que se encarga de capturar y manejar los eventos que genera el dispositivo Emotiv, el que encapsula los valores que se obtienen y genera un nuevo evento, para que sea manejado por las cinco opciones de movimiento que tendrá el robot (que serán descritas más adelante), las que analizarán si el evento generado corresponde a la acción asociada y en ese caso ejecutar el movimiento del robot.

\begin{tabular}{|c|c|c|}
\hline $\begin{array}{l}\text { NXTB } \\
\text { Clase }\end{array}$ & & 7 \\
\hline \multicolumn{3}{|c|}{$\Xi$ Propiedades } \\
\hline \multicolumn{3}{|c|}{ IsConnected } \\
\hline \multicolumn{3}{|c|}{$\Xi$ Métodos } \\
\hline$\theta$ & Cont & \\
\hline & Dise & \\
\hline & NXT & \\
\hline & Play & \\
\hline$\theta$ & SetN & \\
\hline \multicolumn{3}{|c|}{$\boxplus$ Eventos } \\
\hline \multicolumn{3}{|c|}{$\Psi$ Tipos anidados } \\
\hline \multirow{2}{*}{\multicolumn{2}{|c|}{$\begin{array}{l}\text { Robot } \\
\text { Clase } \\
\rightarrow \text { Form }\end{array}$}} & A \\
\hline & \multicolumn{2}{|c|}{$\Phi$ Campos } \\
\hline \multicolumn{3}{|c|}{ E Métodos } \\
\hline \multicolumn{2}{|c|}{$\oplus_{\mathrm{a}}$ emotiv_ActualizacionEvento } & $\oplus_{\mathrm{a}}$ emotiv_Conectado \\
\hline \multicolumn{3}{|c|}{$0_{\text {a }}$ emotiv_Desconectado } \\
\hline \multicolumn{3}{|c|}{$\oplus_{\mathrm{a}}$ EventoAvanzar } \\
\hline \multicolumn{3}{|c|}{ Q. EventoDerecha } \\
\hline \multicolumn{3}{|c|}{ Eventolzquierda } \\
\hline \multicolumn{3}{|c|}{$P_{a}$ EventoParar } \\
\hline \multicolumn{3}{|c|}{$Q_{0}$ EventoRetroceder } \\
\hline \multicolumn{3}{|c|}{$\mathscr{P}_{\mathrm{a}}$ InicializarEmotiv } \\
\hline \multicolumn{3}{|c|}{$\varphi_{a}$ Inicializarlnterfaz } \\
\hline \multicolumn{3}{|c|}{$\oplus_{\mathrm{a}}$ InicializarRobot } \\
\hline \multicolumn{3}{|c|}{$\varphi_{\mathrm{a}}$ InicializarVariables } \\
\hline \multicolumn{3}{|c|}{$\oplus_{\star}$ OnEventoEmotiv } \\
\hline \multicolumn{3}{|c|}{$Q_{\star}$ procesarEvento } \\
\hline \multicolumn{3}{|c|}{ Robot } \\
\hline \multicolumn{3}{|c|}{$\Xi$ Eventos } \\
\hline \multicolumn{3}{|c|}{$5_{\mathrm{a}}$ EventoEmotiv } \\
\hline \multicolumn{3}{|c|}{ \pm Tipos anidados } \\
\hline
\end{tabular}

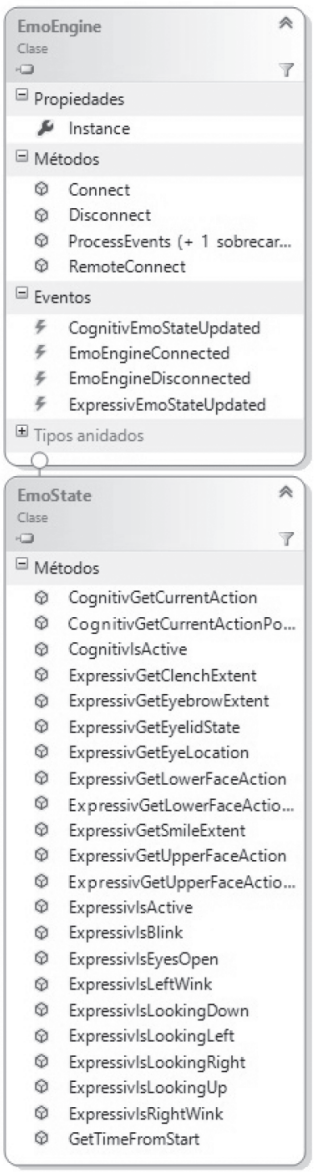

Figura 5. Diagrama de clases.

\section{DESARROLLO DEL SISTEMA}

El sistema considera cinco acciones de movimiento disponible, que son avanzar, ir a la izquierda, ir a la derecha, retroceder y parar, estas se activan cuando se genera un evento expresivo o cognitivo que corresponda a la propiedad seleccionada para cada acción.

Por ejemplo, si el usuario realiza un pestañeo y está asociado a la acción avanzar, el robot encenderá sus motores (en caso de que estén apagados) y avanzará indefinidamente hasta que ocurra otra acción que le haga cambiar de estado. En el caso del gesto mirar a la derecha asociado a la acción de ir a la derecha, no es necesario mantener el gesto para que el robot realice esta acción, simplemente con ejecutarla una 


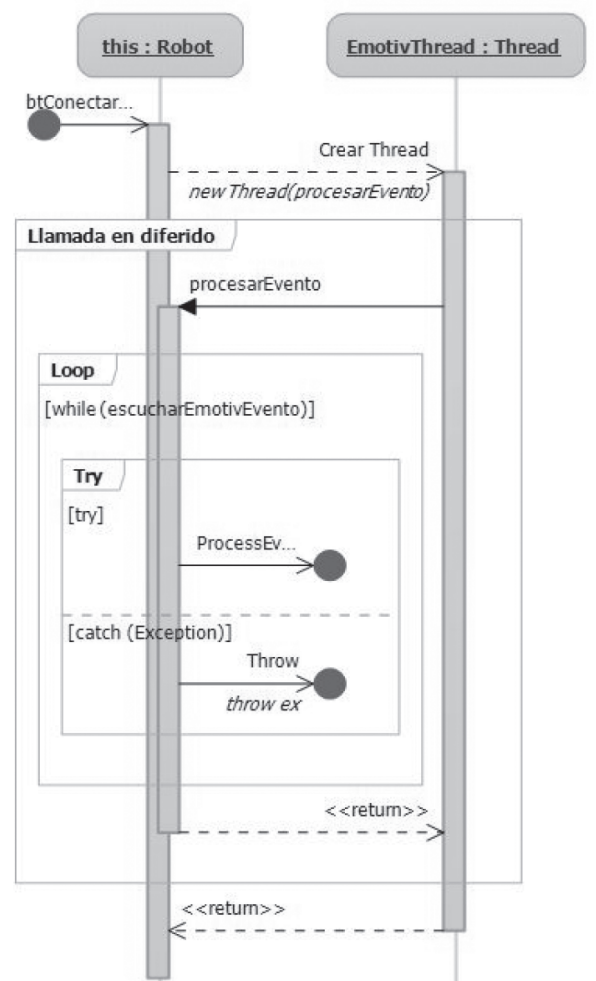

Figura 6. Diagrama de secuencia de conexión y procesamiento.

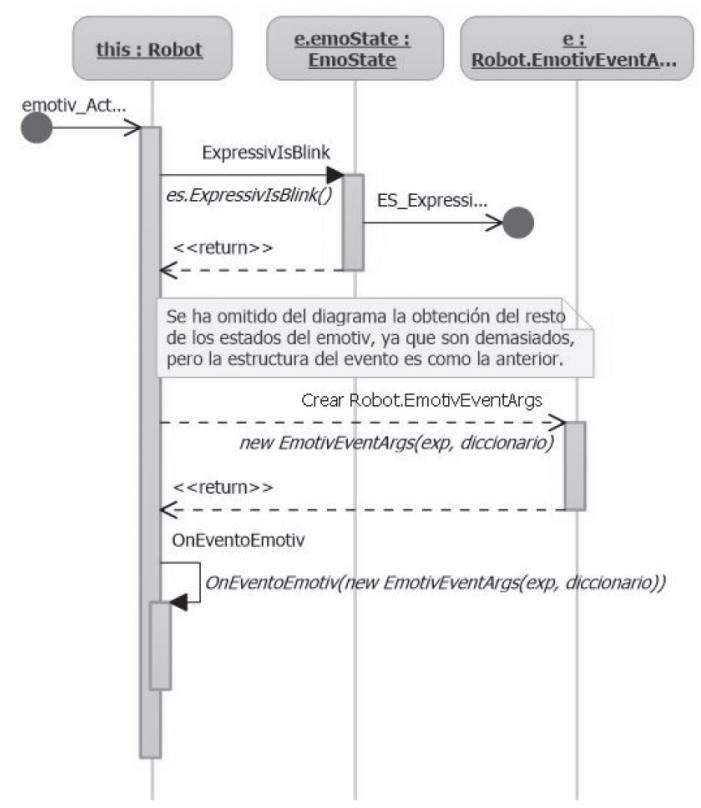

Figura 7. Diagrama de secuencia para manejar evento. vez basta, en otras palabras el usuario puede mirar a la derecha y luego volver a mirar al frente y el robot ira a la derecha indefinidamente hasta que se ejecute otra acción.

En la Figura 8 se muestra el diagrama de flujo sobre la secuencia a seguir entre eventos y acciones diseñadas por medio de la arquitectura dirigida por eventos.

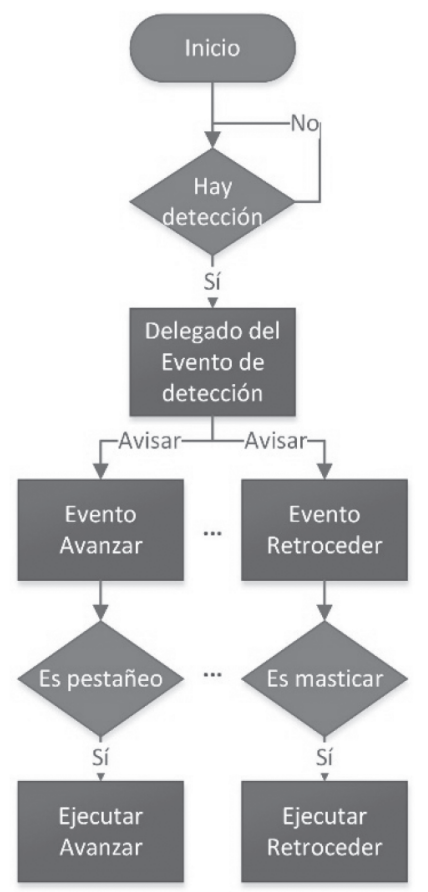

Figura 8. Diagrama de flujo de eventos.

En la Figura 9 se aprecia la interfaz del sistema, en donde el panel de la izquierda está el área de selección de acciones y velocidades del robot, y en la derecha están las conexiones con los dispositivos y la activación del sistema.

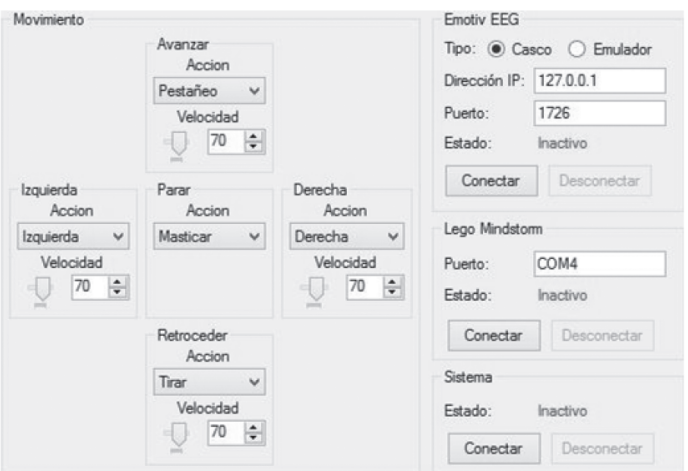

Figura 9. Sistema de movimiento. 
Cuando se genera un evento Emotiv, este entrega un conjunto de señales correspondientes a las acciones que ocurrieron en ese momento, como por ejemplo, las acciones de pestañeo, guiño y masticar del área expresiva (gestos faciales) y empujar, tirar y rotar del área cognitiva (pensamiento). En la Figura 10 se muestran todas las acciones disponibles a elegir.

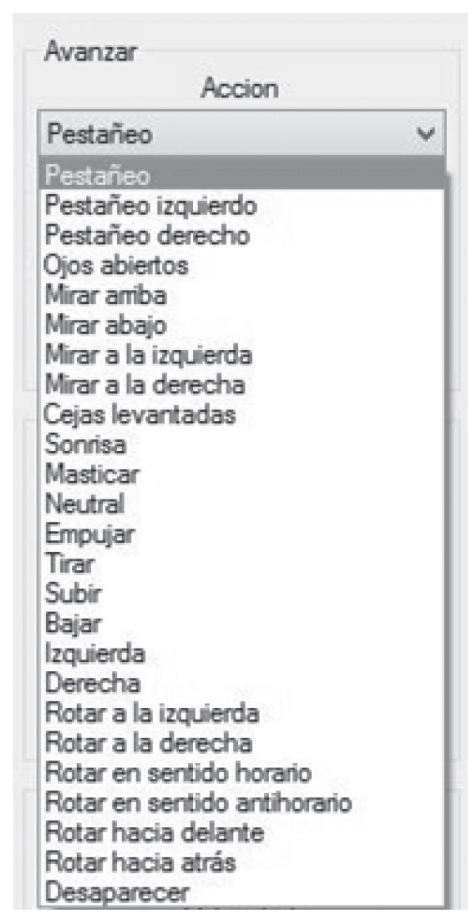

Figura 10. Listado de acciones disponibles.

Además de escoger la acción, se puede elegir la potencia de movimiento del robot, teniendo rangos desde 0 a 100. En la Figura 11 se muestra esta configuración, existiendo la posibilidad de establecer el valor mediante la barra de control deslizante (track bar) o desde el cuadro numérico.

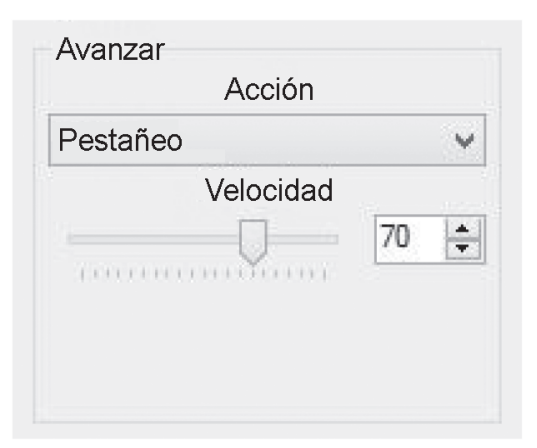

Figura 11. Panel de configuración para la acción avanzar.

\section{PRUEBAS Y RESULTADOS}

Para la utilización de acciones cognitivas se requiere un entrenamiento previo de ello. Se ha fijado un total de dos acciones como máximo (disponible hasta cuatro acciones) para el aprendizaje del pensamiento, ya que con más de dos se requiere un entrenamiento que toma bastante tiempo.

Se ha utilizado a seis sujetos de prueba de sexo masculino y edades entre 24 y 26 años para la realización del entrenamiento. Los resultados mostrados por la Tabla 1 indican el tiempo de entrenamiento y la evaluación de la efectividad del reconocimiento (en porcentaje), respecto de lo que realmente estaban pensando, entregando una precisión de reconocimiento promedio de $81 \%$.

Tabla 1. Tiempos de entrenamiento y porcentaje de reconocimiento.

\begin{tabular}{|c|c|c|}
\hline Sujeto & $\begin{array}{c}\text { Tiempo de } \\
\text { entrenamiento }\end{array}$ & $\begin{array}{c}\text { Porcentaje de } \\
\text { reconocimiento }\end{array}$ \\
\hline 1 & 37 minutos & 80 \\
\hline 2 & 46 minutos & 85 \\
\hline 3 & 53 minutos & 70 \\
\hline 4 & 48 minutos & 75 \\
\hline 5 & 35 minutos & 85 \\
\hline 6 & 40 minutos & 90 \\
\hline
\end{tabular}

En el caso de los gestos faciales, no se requiere entrenamiento previo, ya que las señales son genéricas. Se realizaron pruebas con distintos gestos, en donde los sujetos realizaron 10 repeticiones de cada acción, para posteriormente verificar los niveles de detección. Estos fueron los resultados:

Tabla 2. Porcentaje de reconocimiento de gestos faciales parte 1 .

\begin{tabular}{|c|c|c|c|}
\hline Sujeto & Pestañeo & $\begin{array}{c}\text { Mirar } \\
\text { derecha }\end{array}$ & $\begin{array}{c}\text { Mirar } \\
\text { Izquierda }\end{array}$ \\
\hline 1 & 100 & 100 & 100 \\
\hline 2 & 90 & 100 & 100 \\
\hline 3 & 90 & 80 & 80 \\
\hline 4 & 100 & 90 & 90 \\
\hline 5 & 90 & 70 & 60 \\
\hline 6 & 100 & 70 & 70 \\
\hline
\end{tabular}

En la Tabla 2 se puede observar que las tres primeras acciones tuvieron muy buen nivel de reconocimiento, pero en la Tabla 3 para las acciones de guiño izquierdo y derecho existieron problemas. Esto se 
Tabla 3. Porcentaje de reconocimiento de gestos faciales parte 2 .

\begin{tabular}{|c|c|c|c|}
\hline Sujeto & $\begin{array}{c}\text { Guiño } \\
\text { Derecho }\end{array}$ & $\begin{array}{c}\text { Guiño } \\
\text { Izquierdo }\end{array}$ & Masticar \\
\hline 1 & 20 & 30 & 100 \\
\hline 2 & 20 & 20 & 100 \\
\hline 3 & 0 & 0 & 100 \\
\hline 4 & 10 & 20 & 100 \\
\hline 5 & 30 & 20 & 100 \\
\hline 6 & 40 & 10 & 100 \\
\hline
\end{tabular}

puede deber al nivel de habilidad de la persona para realizar el guiño, la intensidad con que lo realiza, el nivel de conexión del casco con el cuero cabelludo, entre otros factores que podrían impedir una buena detección. Por último la detección de masticar fue de $100 \%$ en todos los casos, por lo que es una acción muy recomendable a usar. El promedio general de detección de las acciones realizadas es de un $67 \%$, pero si descontamos del promedio las acciones de guiño, se obtiene una precisión de $91 \%$, un valor bastante alto, lo que indica que esas acciones entregan muy buenos resultados de reconocimiento.

Indagando en las señales en bruto captadas desde las pruebas realizadas, la señal de guiño derecho en algunos sujetos generó varios picos en la onda del canal F8 como se aprecia en la Figura 12, sin embargo su detección fue bastante pobre, por lo que podría ser un problema de detección del algoritmo del sistema Emotiv. Por otro lado, también se observó que algunos sujetos no eran capaces de realizar un guiño correctamente, por lo que también pudo influir en su detección.

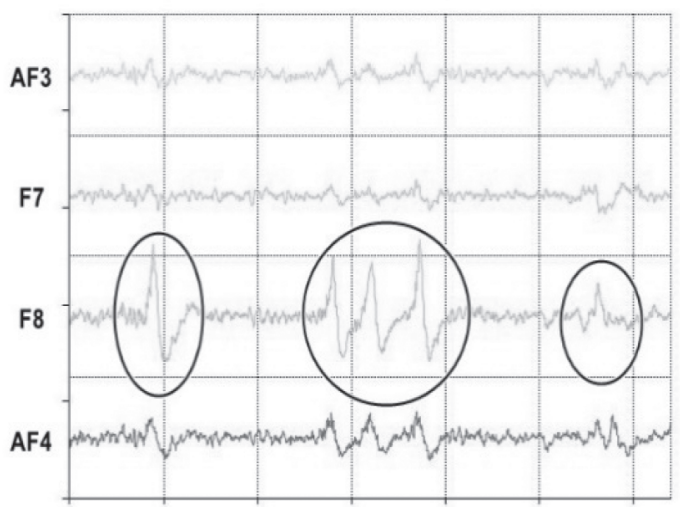

Figura 12. Señal de guiño derecho.
Finalmente, se realizó la prueba de control de movimiento del robot utilizando para ello cinco gestos faciales, que fueron asignados a cada acción de movimiento disponible, los que se detallan en la Tabla 4.

Tabla 4. Asignación de acciones en el sistema.

\begin{tabular}{|l|l|}
\hline Acción & Gesto \\
\hline Avanzar & Pestañeo \\
\hline Derecha & Mirar derecha \\
\hline Izquierda & Mirar izquierda \\
\hline Retroceder & Sonreír \\
\hline Parar & Masticar \\
\hline
\end{tabular}

Los resultados de la prueba indican que entre lo que se desea realizar y lo que se realiza realmente, está en un $70 \%$ de concordancia. Esto es debido a varios factores como se mencionan anteriormente incluyendo ahora la acción de mover la cabeza y hablar que alteran las señales, ya que aún no existen dispositivos EEG que no se vean afectados por los movimientos.

En el link http://youtu.be/H6_m9hq6nkc, se puede ver un video resultante de lo desarrollado en este trabajo. Las Figuras 12, 13, 14 y 15 muestran acciones de las pruebas realizadas con el robot.

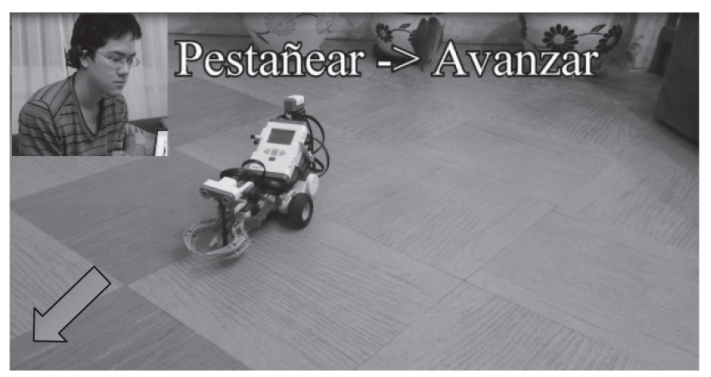

Figura 13. Pestañear para avanzar.

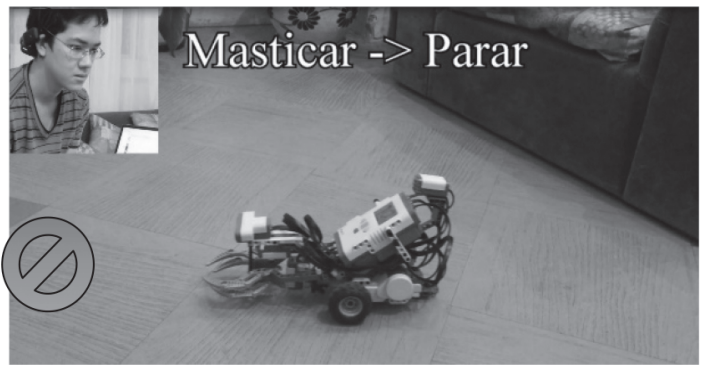

Figura 14. Masticar para detener el robot en movimiento. 


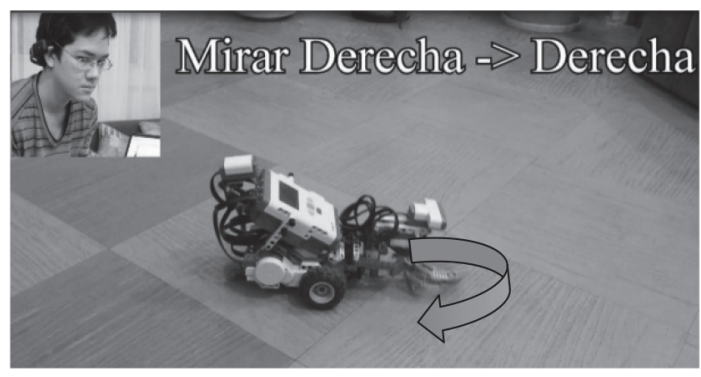

Figura 15. Mirar a la derecha para ir a la derecha parte 1.

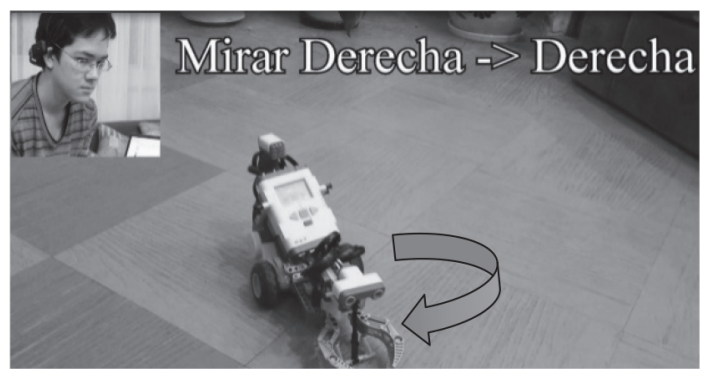

Figura 16. Mirar a la derecha para ir a la derecha parte 2.

\section{CONCLUSIONES}

Inicialmente se puede decir que el SDK de Emotiv posee un buen diseño arquitectónico (orientado a eventos), el que permite capturar la información que realmente se desee utilizar, mediante la suscripción a los eventos correspondientes, como por ejemplo conocer el estado de conexión del dispositivo Emotiv EEG o los eventos cognitivos generados, de los que en vez de preguntar de forma explícita e iterativa por la información, se espera a que el publicador genere un evento para que subsecuentemente se ejecuten las funciones suscritas, manteniendo un buen orden y claridad en el ámbito del código, por lo que hace más legible y escalable la aplicación.

El SDK junto con el dispositivo Emotiv EEG en el caso de acciones expresivas, tiene un buen grado de detección para la mayoría de sus opciones (pestañear, masticar, mirar a los lados, etcétera), obteniendo una precisión promedio de $67 \%$, mejorable sustancialmente si se descuenta del promedio la acción de guiño que no fue detectada correctamente, obteniendo una alta precisión del 91\%. Posiblemente, la pobre detección de la acción de guiño haya sido provocada por dos motivos, una donde el usuario no tiene la habilidad de realizar un guiño correctamente y la otra donde el software de Emotiv no es capaz de reconocerlo.

En el caso del área cognitiva, si bien está limitado hasta cuatro acciones como máximo, ya desde el entrenamiento para la tercera acción se complica bastante, requiriendo un tiempo considerable (más de 1 hora) de entrenamiento que fatiga mentalmente al usuario, por lo que se recomienda utilizar solo dos acciones, ya que su periodo de entrenamiento que ronda por los 43 minutos y su precisión promedio de $81 \%$ están en un rango aceptable y que seguramente con un mayor entrenamiento se obtendrán mejores resultados.

Finalmente, destacar que el dispositivo Emotiv EEG es una buena alternativa a usar en comparación de los sistemas tradicionales de EEG, que cuentan con muchos más canales que Emotiv, generando complicaciones en el posicionamiento correcto de los sensores, además que no son portables ni inalámbricos, y su costo es sumamente elevado, por lo que se concluye que Emotiv es un buen dispositivo EEG respecto de su calidad/precio, ofreciendo varias opciones de reconocimiento con una buena precisión, en comparación con los trabajos descritos anteriormente donde la precisión fue baja, y ofreciendo resultados en tiempo real, comparándolo con el trabajo descrito en [5] que su reconocimiento requería de tiempo que puede ser valioso para el usuario, por lo que en conclusión, Emotiv EEG puede ser utilizado como interfaz para el control de dispositivos.

\section{RECOMENDACIONES}

Los 16 sensores de Emotiv están compuestos de una base con metal y un fieltro, los que deben ser humedecidos con agua salinizada para su uso. Posteriormente, el manual indica que para su almacenamiento, se deben guardar los sensores en su caja de hidratación, la cual incluye una almohadilla que cubre todos los sensores (fieltros), y esta debe ser humedecida con líquido salino.

Debido a esto, con el tiempo se han oxidado los sensores, lo que puede provocar ruidos en las señales y ocasionar problemas en la detección, por lo que se 
recomienda sacar el fieltro de la base, y almacenarlo de forma separada, para evitar la oxidación de este. En la Figura 17 se puede observar cómo han quedado los sensores a causa del almacenamiento húmedo provocado por la utilización del líquido salino luego de múltiples usos.

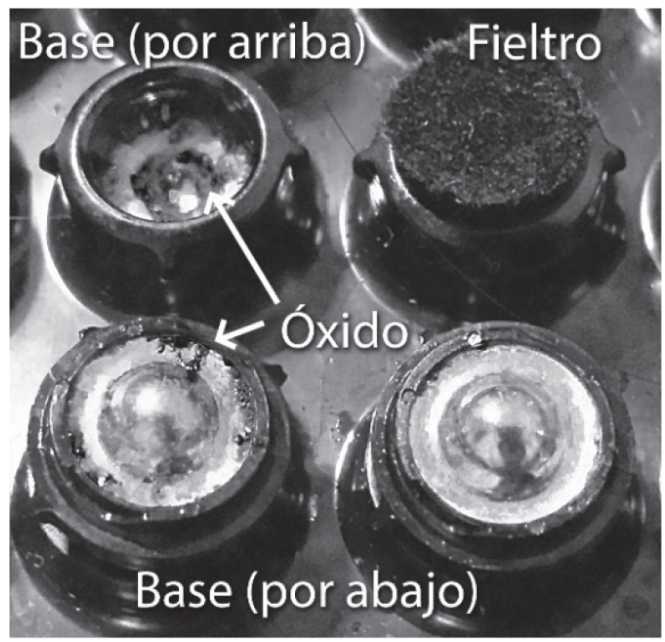

Figura 17. Sensores de Emotiv oxidados.

\section{TRABAJO A FUTURO}

El desarrollo de este proyecto sigue activo, por lo que se espera implementar el sistema por medio de interfaces para las clases, de esta forma será posible realizar cambios de dispositivos de una manera más rápidamente, en el caso del sistema de captura de EEG, que podría pasar de Emotiv a NeuroSky o del dispositivo motor pasando de Lego Mindstorms a una silla eléctrica u otros.

También se está trabajando en un sistema de reconocimiento de patrones, como complemento al sistema de reconocimiento que trae por defecto el API de Emotiv, otorgando distintos mecanismos de detección que se adecuen mejor al usuario.

\section{AGRADECIMIENTOS}

Agradecer en parte al financiamiento aportado por el proyecto de pregrado 8711-14 denominado "Sistema de Control Mediante Señales Neurológicas para Gobernar Movimientos" Universidad de Tarapacá, $\mathrm{y}$ a todas las pacientes personas que entregaron parte de su tiempo para realizar las pruebas con el dispositivo.

\section{REFERENCIAS}

[1] H. Wisniowski. “Analog Devices And Nintendo Collaboration Drives Video Game Innovation With iMEMS Motion Signal Processing Technology". Norwood MA, United States. 2006.

[2] Microsoft. "Project Natal". 2009. Date of visit: June 10, 2014. URL: http://blog. seattlepi.com/digitaljoystick/2009/06/01/ e3-2009-microsoft-at-e3-several-metric-tonsof-press-releaseapalloza/

[3] Emotiv. "EEG Specifications". 2012. Date of visit: June 12, 2014. URL: http://emotiv. com/upload/manual/EEGSpecifications.pdf

[4] J. O'Connor. "Real-time Control of a Robot Arm Using an Inexpensive System for Electroencephalography Aided by Artificial Intelligence". Computer Science Honors Papers. No 3. 2013.

[5] M. Palankar, K. De Laurentis and R. Dubey. "Using biological approaches for the control of a 9-DoF wheelchair-mounted robotic arm system: Initial experiments". IEEE International Conference on Robotics and Biomimetics, pp. 1704-1709. February, 2009.

[6] A. Vourvopoulos and F. Liarokapis. "Braincontrolled NXT Robot: Tele-operating a robot through brain electrical activity". Third International Conference on Games and Virtual Worlds for Serious Applications, pp. 140-143. 2011.

[7] J. Esteban, J. Cárdenas y A. Peña. "Sistema para Rehabilitación del Síndrome del Miembro Fantasma utilizando Interfaz CerebroComputador y Realidad Aumentada". Revista Ibérica de Sistemas y Tecnologías de Información. № 11, pp. 93-106. Junio 2013.

[8] American Electroencephalographic Society. "Guideline thirteen: Guidelines for standard electrode position nomenclature". Journal of Clinical Neurophysiology, pp. 25:111-113. 1994.

[9] AForge.NET. "AForge.NET". Date of visit: June 14, 2014. URL: http://www.aforgenet. $\mathrm{com} /$

[10] TheLEGOGroup. “LEGO®MINDSTORMS® NXT Executable File Specification”. 2009. Date of visit: June 14, 2014. URL: http:// www.lego.com/es-ar/mindstorms/downloads/ nxt/nxt-sdk 